\title{
UV, visible and IR active media based on scheelite-type and colquirite-type fluoride crystals
}

Vadim Semashko, Alexander Naumov, Alexey Nizamutdinov, Stella Korableva, Michail Marisov and Alexey Shavelev

Kazan Federal University, 420008 Kazan, Russia

\begin{abstract}
The experimental results of applying crystal-chemical methods aimed to improving the spectral-kinetic properties of active media based on fluoride crystals with scheelite $\left(\mathrm{LiY}_{1-\mathrm{x}} \mathrm{LuF}_{4}\right)$ and colquiriite $\left(\mathrm{LiCa}_{1-\mathrm{x}} \mathrm{AlF}_{6}\right)$ structures doped by rare-earth $\left(\mathrm{Ce}^{3+}\right.$ and $\left.\mathrm{Pr}^{3+}\right)$ and $\mathrm{Cr}^{3+}$ ions are discussed. The energetic and spectral characteristics of lasers based on the active media are presented. The special attention is focused on the prospects of using these lasers in the task of optosensorics, biomedicine, and security systems.
\end{abstract}

Fluoride crystalline active media allow realizing laser action in an unprecedentedly wide range of wavelengths, ranging from medium IR to vacuum ultraviolet. This opportunity appears due to the widest band gap of fluoride hosts among other known crystalline compounds and a relatively narrow phonon spectrum [1]. The latter circumstance also contributes to obtaining laser oscillations at transitions of impurity ions, which are difficult to use in other materials because off intense nonradiative processes and the followed low quantum yield. In addition, crystalline fluorides have good thermo-optical properties, mechanical strength and radiation resistance, which allow to design powerful laser systems with the $\mathrm{M}^{2}$ coefficient closing to unity under relatively simple technical solutions.

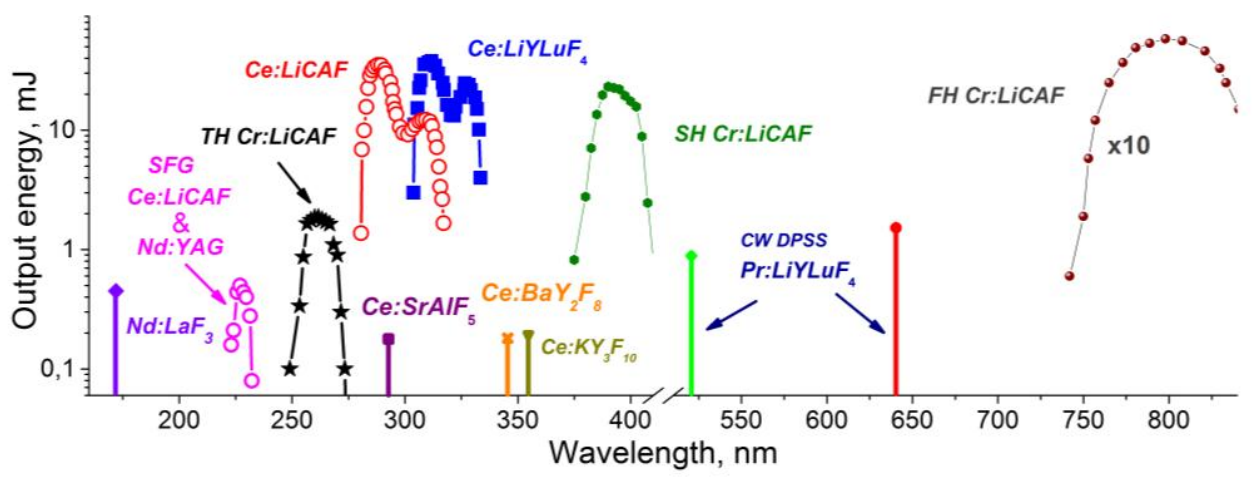

Fig. 1. Summary properties of solid-state lasers based on fluoride crystals with the structure of scheelite $\left(\mathrm{LiY}_{1-\mathrm{x}} \mathrm{Lu}_{\mathrm{x}} \mathrm{F}_{4}\right)$ and colquiriite $\left(\mathrm{LiCa}_{1-\mathrm{x}} \mathrm{Sr}_{\mathrm{x}} \mathrm{AlF}_{6}\right)$, implemented at Kazan Federal University. 
Ultraviolet tunable laser radiation can be obtained either using $4 \mathrm{f}^{\mathrm{n}-1} 5 \mathrm{~d}-4 \mathrm{f}^{\mathrm{n}}$ dipole-allowed interconfiguration transitions of trivalent rare-earth ions (REI) in wide-band-gap crystals [2], or by non-linear frequency conversion of the radiation of IR lasers operating on transitions of iron group ions [3]. In the first case, radiation from other lasers is used for pumping, and in the second, to do that can be successfully applied a radiation from lamps or laser diodes. Efficient narrow-band laser action of the visible range is currently realized on $4 \mathrm{f}-4 \mathrm{f}$ transitions of $\mathrm{Pr}^{3+}, \mathrm{Sm}^{3+}, \mathrm{Tb}^{3+}, \mathrm{Dy}^{3+}, \mathrm{Ho}^{3+}$ and $\mathrm{Er}^{3+}$ dopants in crystals pumped by $\mathrm{InGaN}$ (GaN) laser diode radiation (DPSS lasers) [4].

The report provides an overview of many years of researches results conducted at the Kazan Federal University, aimed to search for new solid-state active media of the UV, visible and IR ranges. In particular, the experimental results of applying crystal-chemical methods improving the spectral-kinetic properties of active media based on fluoride crystals with the structure of scheelite $\left(\mathrm{LiY}_{1-\mathrm{x}} \mathrm{Lu}_{\mathrm{x}} \mathrm{F}_{4}\right)$ and colquiriite $\left(\mathrm{LiCa}_{1-\mathrm{x}} \mathrm{Sr}_{\mathrm{x}} \mathrm{AlF}_{6}\right)$ hosts doped by $\mathrm{Ce}^{3+}, \mathrm{Pr}^{3+}$ and $\mathrm{Cr}^{3+}$ are discussed. The output and spectral properties of lasers based on these crystals are presented in Fig.1. The negative impact of the photodynamic processes induced by pumping radiation into solid-state active media on laser properties is analyzed, and the approaches minimizing their consequences are proposed. Particular attention is directed to the prospects of using solid-state lasers based on the studied fluoride crystals for biomedicine applications, optosensorics and security systems.

The study was done in the frame of the subsidy allocated to KFU for the state assignment in the sphere of scientific activities (3.1156.2017/4.6). V.V. Semashko personally thanks the project 3.6722.2017/8.9 (former 3.5835.2017/6.7)) for the financial support.

\section{References}

1. A. A. Blistanov, V. S. Bondarenko, N. V. Perelomova, F. N. Strizhevskaya, V. V. Chkalova, M. P. Shaskol'skaya, Acoustical Crystals (Nauka, Moskow, 1982) (in Russian)

2. V. V. Semashko, Phys. of Sol. State., 47, 1507 (2005)

3. J. F. Pinto, L. Esterowitz, G. H. Rosenblatt, IEEE J. Sel. Top. Quantum Electron., 1, 58 (1995)

4. C. Krankel, D.-T. Marzahl, F. Moglia, G. Huber, P.W. Metz, Laser Photonics Rev., 10, 548 (2016) 